\title{
A Comprehensive Survey of Genomic Alterations in Gastric Cancer Reveals Recurrent Neoantigens as Potential Therapeutic Targets
}

\author{
Chao Chen ${ }^{(1)},{ }^{1,2,3}$ Qiming Zhou, ${ }^{3,4}$ Riping Wu, ${ }^{5}$ Bo Li, ${ }^{2,3}$ Qiang Chen, ${ }^{5,6,7}$ \\ Xiuqing Zhang, ${ }^{1,2,3}$ and Chunmei Shi $\mathbb{D}^{5,6}$ \\ ${ }^{1}$ BGI Education Center, University of Chinese Academy of Sciences, Shenzhen 518083, China \\ ${ }^{2}$ BGI-Shenzhen, Shenzhen 518083, China \\ ${ }^{3}$ BGI-GenoImmune, Wuhan 430079, China \\ ${ }^{4}$ Hospital of Shenzhen Nanshan District, Shenzhen 518052, China \\ ${ }^{5}$ Fujian Medical University Union Hospital, Fuzhou 350001, China \\ ${ }^{6}$ Fujian Provincial Key Laboratory of Translational Cancer Medicine, Fuzhou 350004, China \\ ${ }^{7}$ Fujian Medical University Stem Cell Research Institute, Fuzhou 350004, China
}

Correspondence should be addressed to Chao Chen; 409985846@qq.com and Chunmei Shi; scmfz@qq.com

Received 14 August 2019; Revised 28 September 2019; Accepted 30 September 2019; Published 5 November 2019

Academic Editor: Brandi L. Cantarel

Copyright (c) 2019 Chao Chen et al. This is an open access article distributed under the Creative Commons Attribution License, which permits unrestricted use, distribution, and reproduction in any medium, provided the original work is properly cited.

\begin{abstract}
Immunotherapy directed against cancer-specific neoantigens derived from non-silent mutants is a promising individualized strategy for cancer treatment. Neoantigens shared across patients could be used as a public resource for developing T cell-based therapy. To identify potential public neoantigens for therapy in gastric cancer (GC), 74 GC patients were enrolled in this study. Combined with the TCGA cohort and other published studies, whole exome sequencing data from $942 \mathrm{GC}$ patients were used to detect somatic mutations and predict neoantigens shared by GC patients. The mutations pattern between our study and the TCGA cohort is comparable, and $\mathrm{C}>\mathrm{T}$ is the most common substitution. The number of neoantigens was significantly higher in older patients (age $\geq 60$ ) compared to younger patients (age $<60$ ), both in this study and the TCGA cohort. Recurrent neoantigens were found in eight genes (TP53, PIK3CA, PGM5, ERBB3, C6, TRIM49C, OR4C16, and KRAS) in this study. The neoantigen-associated mutations PIK3CA (p.H1047R) and TP53 (p.R175H) are common across several cancer types, indicating their potential usage. Overall, our study illustrates a comprehensive genomic landscape of GC and provides the recurrent neoantigens to facilitate further immunotherapy.
\end{abstract}

\section{Introduction}

Gastric cancer (GC) is the leading cause of cancer-related death around the world and is particularly prevalent in east Asian countries $[1,2]$. Chemotherapy and radiotherapy are the traditional treatment methods for GC. Despite the enhancement in eradication of Helicobacter pylori and early cancer screening, the 5-year overall survival rate for GC worldwide is still below 30\% [3].

With the development of biologics, immunotherapy has revolutionized oncology by targeting the host immune system. There are many ways of immunotherapy, such as immune checkpoint inhibitors (ICIs), cancer vaccine, and mutant-antigen-specific tumor-infiltrating lymphocytes (TILs) [1, 4-7]. Recent studies have highlighted the role of tumor-specific neoantigens in cancer immunotherapy, promoting personalized vaccines and cell therapies based on cancer somatic mutations $[8,9]$. Several studies have used neoantigens as targets in $\mathrm{T}$ cell-based immunotherapy for treatment of melanoma $[10,11]$, malignant gliomas [12], ovarian cancer [13], and breast cancer [4]. Although neoantigen-based immunotherapy had been used in many cancers, they have not been used in GC as far as we know. 
The emergence of next-generation sequencing technology has rapidly expanded our understanding of the genetic basis of GC, and many studies have provided useful crossvalidation information [14-20]. However, most of these studies focused on the mechanism of gene mutation and tumorigenesis but did not involve the neoantigen landscape of GC, and the sample size of a single study was limited. Zhou and colleagues have analyzed the neoantigens in 32 patients with GC. However, due to the limitations of sample size and detection methods, the study did not find neoantigens shared among GC patients [21]. Therefore, in order to promote the immunotherapy of GC based on neoantigens, we combined 74 samples of this study with published sequencing data to obtain 942 samples and carried out the largest study of the neoantigen landscape of GC so far.

\section{Materials and Methods}

2.1. Genomic Data of GC. This study was approved by the Ethical Committee of the Union Medical College Hospital Affiliated of Fujian Medical University and carried out in accordance with the approved guidelines. All patients signed informed consent before admission. In total, samples of cancer and adjacent normal tissues from 75 cases with clinical pathological information were provided, and 74 of them (hereafter referred to as Fujian cohort) passed the quality control for library construction and sequencing (Table 1). Besides, all somatic mutations, including single nucleotide substitutions and short insertion/deletions (indels), from the most recent publications (Table 1 and Supplementary Tables S1-S2), representing the other six geographically different research cohorts were downloaded, and the mutation data of 942 patients were eventually obtained for integrated analysis.

\subsection{Pipeline for Somatic Mutation Analysis and Neoantigen} Prediction. The sequencing data of Fujian cohort were processed with SOAPnuke [22] and mapped to human hg19 reference using Edico (http://edicogenome.com/dragenbioit-platform), and then MuTect [23] and Varscan2 [24] were used to detect the somatic snvs and indels, respectively. Mutations with frequency $>0.5 \%$ in common mutation databases (1,000 genomes database, Exome Sequencing Project 6500 database, dbSNP database, and Exome Aggregation Consortium database) were filtered. The final mutations were annotated with ANNOVAR [25] and transformed to MAF format using Maftools [26] for further statistical analysis and visualization. Some mutation sites were selected for mass spectrometry verification, and the validation rate was $94 \%$ [27].

The HLA genotyping of the Fujian cohort was processed using Polysolver [28], with the previous mapping bam file as input. Data of 408 TCGA samples containing HLA information were downloaded from the dbGAP database. Then, nonsilent snvs in Fujian $(n=74)$ and TCGA cohorts $(n=408)$ were used to predict neoantigens by NetMHC [29], NetMHCpan [30], PickPocket [31], PSSMHCpan [32], and SMM [33]. Peptides need to satisfy the following three
TABLE 1: Summary of clinical information of Fujian cohort, TCGA cohort, and 942 integrated GC samples.

\begin{tabular}{|c|c|c|c|}
\hline Characteristics & Fujian & TCGA & All \\
\hline \multicolumn{4}{|l|}{ Age } \\
\hline$<60$ & 28 & 132 & 318 \\
\hline$\geqq 60$ & 46 & 306 & 589 \\
\hline NA & 0 & 5 & 35 \\
\hline \multicolumn{4}{|l|}{ Sex } \\
\hline Male & 52 & 285 & 607 \\
\hline Female & 22 & 158 & 315 \\
\hline NA & 0 & 0 & 20 \\
\hline \multicolumn{4}{|l|}{ Lauren's type } \\
\hline Intestinal & 32 & 191 & 429 \\
\hline Diffuse & 28 & 72 & 305 \\
\hline Mixed & 10 & 0 & 24 \\
\hline NA & 4 & 180 & 184 \\
\hline \multicolumn{4}{|l|}{ Tumor stage } \\
\hline Stage I & 7 & 59 & 96 \\
\hline Stage II & 7 & 130 & 200 \\
\hline Stage III & 51 & 183 & 418 \\
\hline Stage IV & 9 & 44 & 178 \\
\hline $\mathrm{NA}$ & 0 & 27 & 50 \\
\hline \multicolumn{4}{|l|}{ Differentiation } \\
\hline Poor & 51 & - & 136 \\
\hline Well & 20 & - & 21 \\
\hline Moderate & 0 & - & 32 \\
\hline NA & 3 & 443 & 753 \\
\hline \multicolumn{4}{|l|}{ Location } \\
\hline Antrum & 17 & 162 & 346 \\
\hline Body & 31 & 152 & 286 \\
\hline Cardia & 22 & 62 & 175 \\
\hline Others & 4 & 46 & 59 \\
\hline NA & 0 & 21 & 76 \\
\hline
\end{tabular}

NA, not available.

criteria: (1) length between 8 and 11 mers; (2) affinity IC50 $<500 \mathrm{nM}$ in at least two tools; (3) and the affinity score in mutation-type (MT) peptide is less than that in wild-type (WT) peptide.

2.3. Statistical Analysis. Statistical analyses were carried out by $\mathrm{R}$ studio, and the significance was determined by the Wilcoxon rank sum test or Fisher's exact test, when appropriate. When $p<0.05$, it is defined as significant.

\section{Results}

3.1. Integrated Mutation Landscape of GC Patients. The mutations of GC in Fujian cohort were identified, and the landscape is shown in Figure 1. Totally, there are 10,607 somatic snvs and 511 somatic indels (insertions and deletions), with a median of 66 mutations across samples. $\mathrm{C}>\mathrm{T}$ transition is the main type of mutations, consistent with TCGA and previous reports $[19,34]$. The nonsilent snvs, including 6,857 missense mutations, 463 nonsense mutations, 9 nonstop mutations, and 212 mutations in the splice site were used to predict neoantigens.

For integrated analysis of the 942 GC samples, TP53, TTN, MUC16, LRP1B, SYNE1, and CSMD3 are the most 

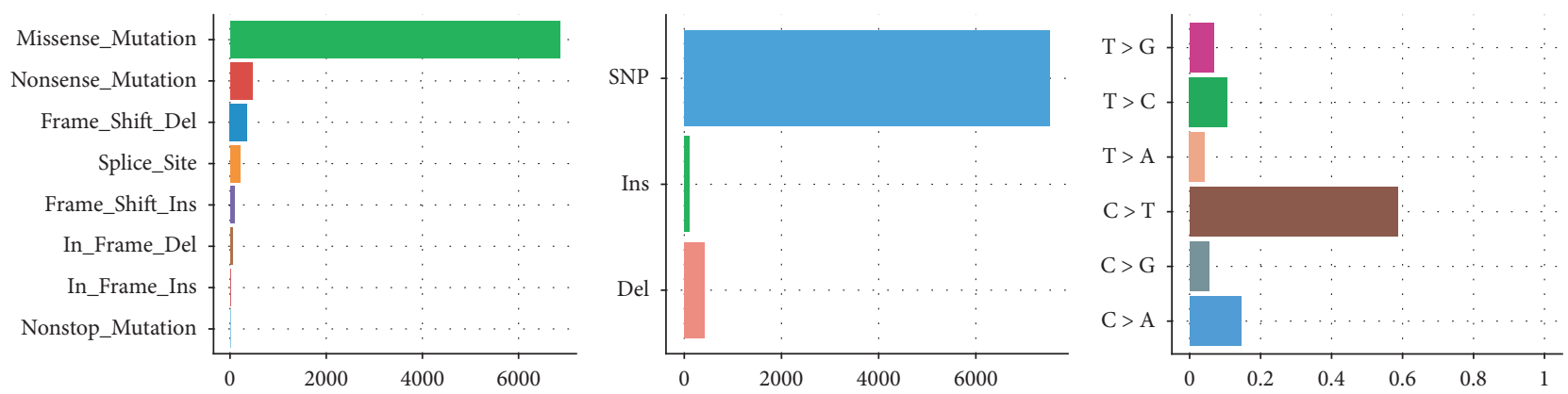

(a)
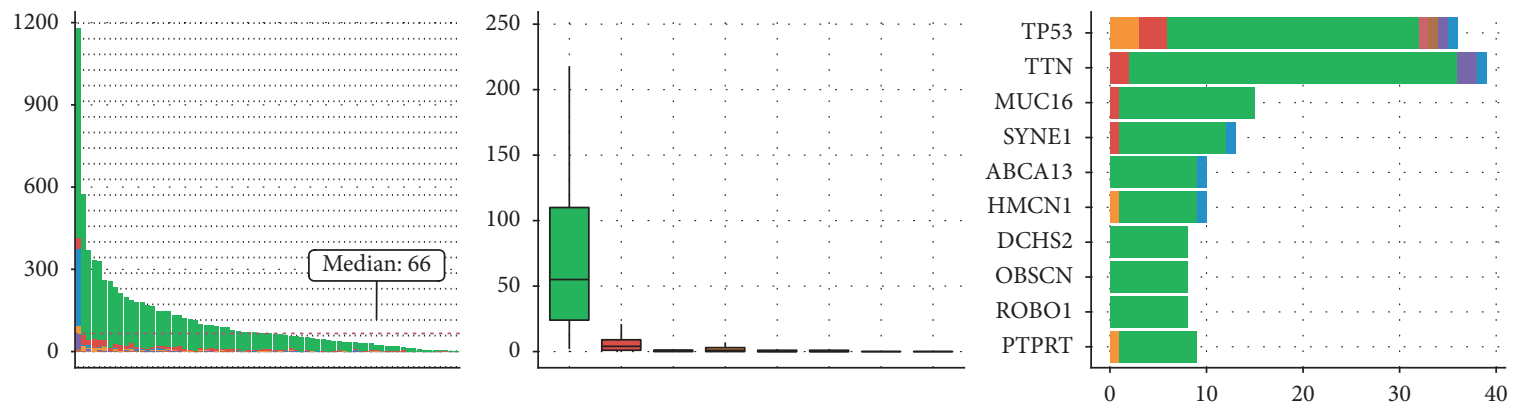

(b)

FIgURE 1: Mutation landscape in Fujian cohort. (a) From left to right, counts of each variant classification, counts of each variant type, and counts of each SNV class are presented. (b) From left to right, variants number per sample, variant classification, and top 10 significantly mutated genes are presented.

frequently mutated genes (Figure S1). Several other cancer driver genes, such as ARID1A, FAT4, and PIK3CA, were also found to mutated in more than $10 \%$ of GC samples. Interestingly, we found five genes were mutated exclusively with TP53, including CDH1, KMT2D, RYR1, PIK3CA, and ARID1A (Fisher's exact test, $P<0.05$, Figure S2). The inverse relationship between ARID1A and TP53 was reported previously [35], which indicates different cancer drive mechanisms. CDH1, KMT2D, RYR1, and PIK3CA were also concordantly mutated with ARID1A (Fisher's exact test, $P<0.05$, Figure S2), suggesting that the carcinogenic mechanism of GC samples that carry mutations in these genes is similar but different from that of samples carrying TP53 mutation.

3.2. Neoantigen Profiling of GC Patients. To explore the neoantigen profiling of GC, we predict the neoantigens for Fujian cohort and TCGA cohort using the nonsilent point mutations, separately. The number of neoantigens in Fujian cohort ranged from 0 to 753 , with a median of 76 . A total of 408 GC samples from TCGA were used to predict neoantigens. The number of neoantigens ranged from 2 to 15268 , with a median of 193 . The reason why the number of neoantigens in TCGA is more than that in Fujian cohort may be that the samples contain more mutations.

Next, we want to know whether the significantly mutated genes (SMGs) also carry more neoantigens. The results show that the SMGs can produce more neoantigens (Figure 2(a)), such as TP53, TTN, MUC16, and ARID1A, which indicates that these genes may carry potential tumor targeting sites.
TP53, which had non-silent mutations in 212 (48.5\%) TCGA samples and 35 (47.3\%) Fujian samples, respectively, produced the largest number of neoantigens in the two cohorts, with 89 and 13 samples carried neoantigens, respectively. MUC16 is a gene that is positively related to the mutation load of tumors and can encode the tumor antigen CA-125. It is believed that the mutation of MUC16 is associated with better prognosis [3]. In the two cohorts of this study, the gene MUC16 also produced many neoantigens.

We further compared the correlation between the number of neoantigens and non-silent mutations in TCGA and Fujian cohorts. The results showed that, in both cohorts, the number of neoantigens was significantly correlated with the number of non-silent mutations, and the Spearman correlation coefficients were $0.92 \quad(P<0.01)$ and 0.88 $(P<0.01)$, respectively (Figures $2(\mathrm{~b})$ and $2(\mathrm{c}))$.

3.3. Comparison of Neoantigens in Different Subtypes and Cohorts of GC. We then grouped the samples according to age, sex, Lauren type, stage, and location of occurrence, and counted the differences in the number of neoantigens between different subgroups. We found that patients older than 60 carried more neoantigens than patients younger than 60 (Wilcoxon rank sum test, $P=0.01$ ). Males tended to carry more neoantigens than females, but not statistically significant (Wilcoxon rank sum test, $P=0.077$, Figures 3 (a) and (b)). There was no significant difference in the neoantigen load between different Lauren types, stages, and different locations (Figures 3(c)-3(e)). The same trend was observed in TCGA samples. 


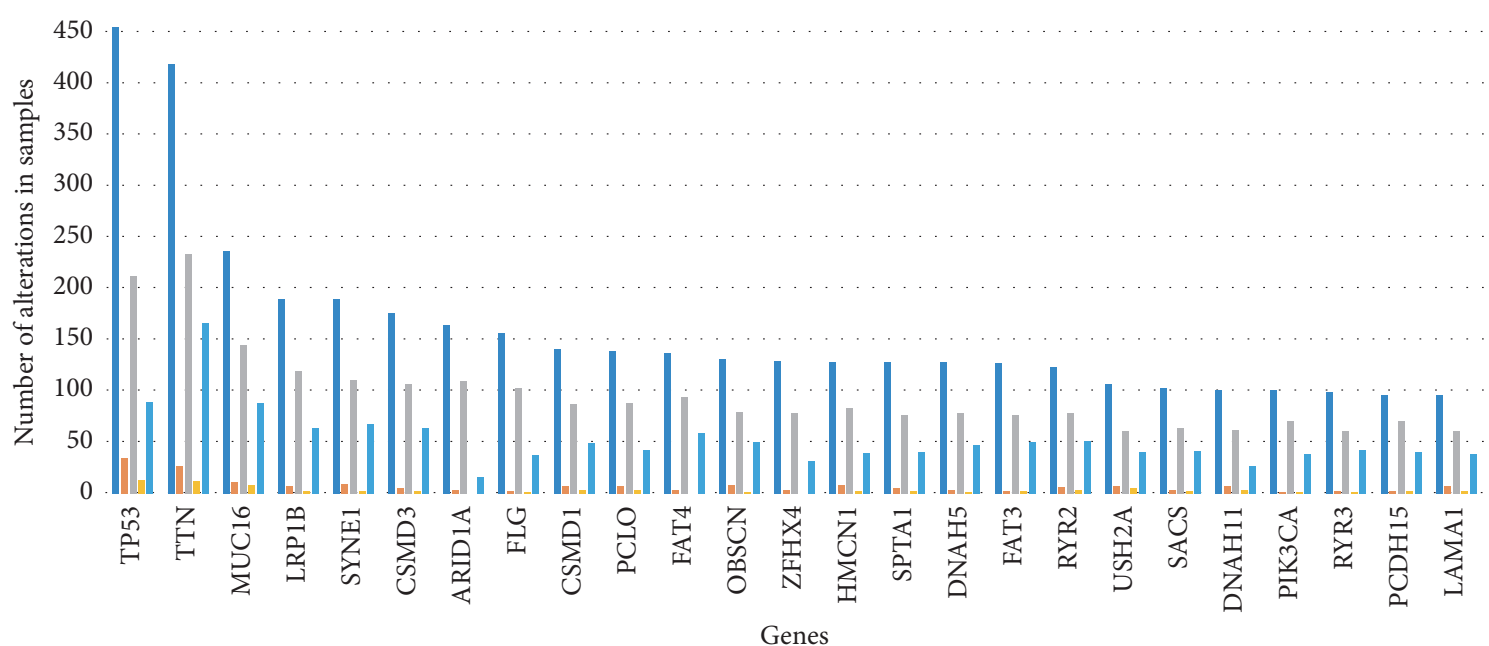

$$
\begin{aligned}
& \text { MutFreqALL } \\
& - \text { MutFreqFuJian } \\
& \text { MutFreqTCGA }
\end{aligned}
$$

(a)

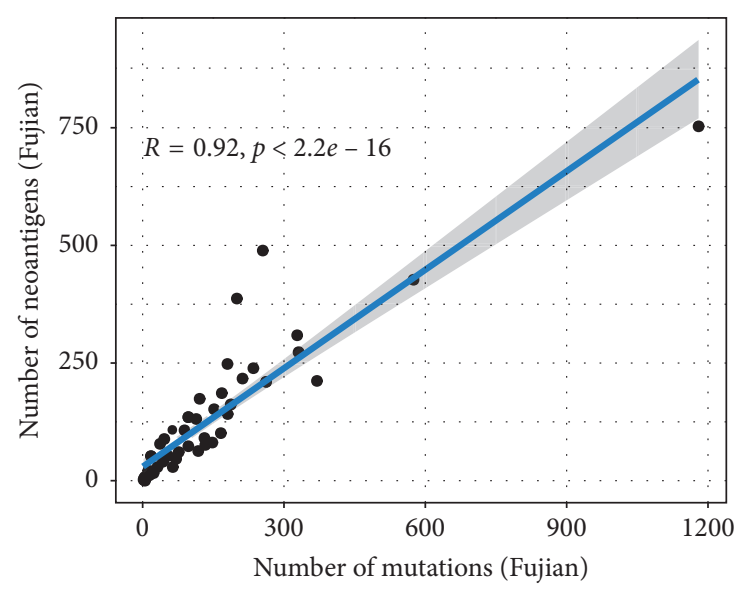

(b)

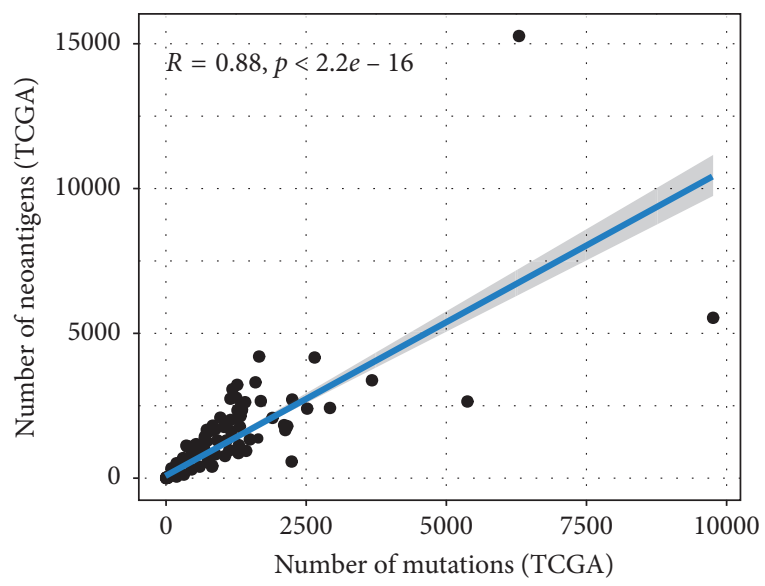

(c)

Figure 2: The overall number of somatic mutations and neoantigens in Fujian and TCGA cohorts. (a) The frequency of mutated genes and their neoantigens in Fujian and TCGA and all GC samples in this study $(n=942)$. The fitted curve between the number of nonsilent somatic mutations and neoantigens in Fujian cohort (b), $R^{2}=0.92$, and TCGA cohort (c), $R^{2}=0.88$, respectively.

Zhou et al. found that 54 genes could produce neoantigens in at least three samples in the Zhejiang cohort [21]. In the Fujian and TCGA cohorts, 2,855 and 15,791 genes carrying neoantigens were detected, and 73 and 9480 genes were found in at least three samples, respectively. Comparing the three cohorts, we found that most of the genes could be covered by the TCGA and Fujian cohorts (Figure 3(f)). At the neoantigen level, no neoantigens shared among GC patients were found in Zhejiang cohort. Two and 486 neoantigens appeared in at least two samples in Fujian and TCGA, respectively. For the reason that the Zhejiang cohort did not find a common neoantigen sequence among GC samples, we speculate that it may be because the size of Zhejiang cohort is too small and they only use NetMHCpan software to predict neoantigens in their research, but the software can only predict a relatively small set of HLA class I alleles [30, 32], so there are some limitations in the prediction of neoantigens.
3.4. Neoantigens Shared among GC Patients. A total of 74,864 neoantigens were detected in Fujian and TCGA samples, of which 549 were found in at least 2 samples (a total of 61 neoantigens were shared between TCGA and Fujian cohorts). The top eight neoantigen associated genes were PGM5, TP53, TRIM49C, PIK3CA, ERBB3, C6, OR4C16, and $K R A S$, respectively (Table 2). If we consider these neoantigens as a panel, it can cover about $15.8 \%$ of the total 482 samples. At present, 10-20 cancer-specific neoantigens are usually synthesized in vitro and used in T cell immunotherapy [11]. Since these neoantigens can cover a certain proportion of GC population, we believe that these recurrent neoantigens of GC have potential clinical application value.

We found that the mutations corresponding to high frequency neoantigens in Fujian and TCGA samples were mostly high frequently mutated sites in all 942 GC samples and TCGA pan-cancer (Table 2, Freq1, Freq2, and Freq3, 

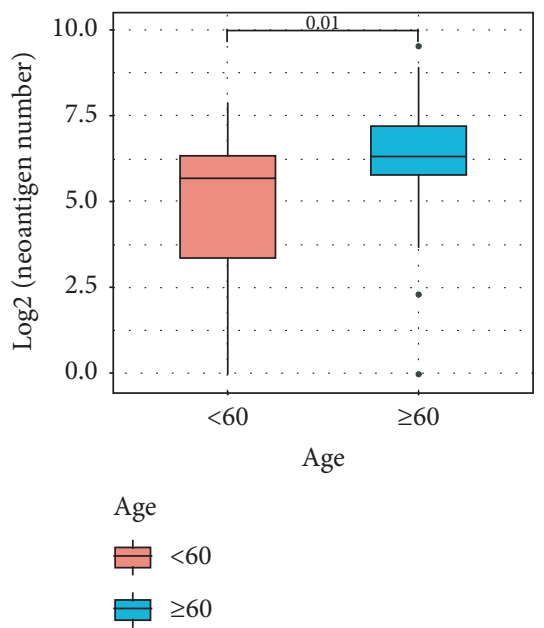

(a)

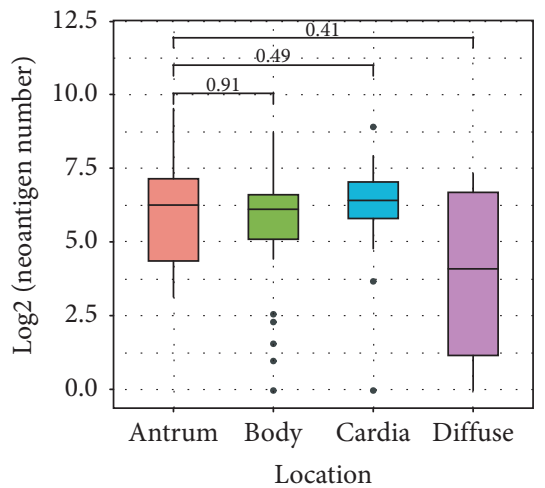

Location

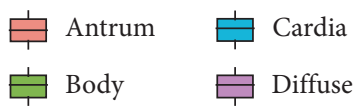

(d)

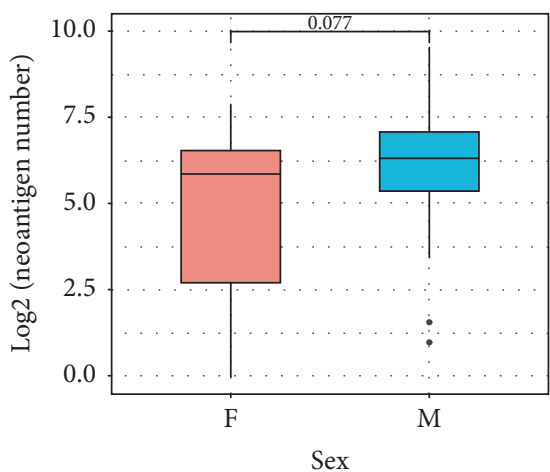

Sex

宁 $\mathrm{F}$

安 $M$

(b)

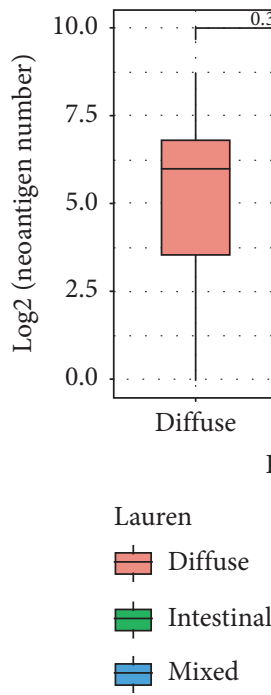

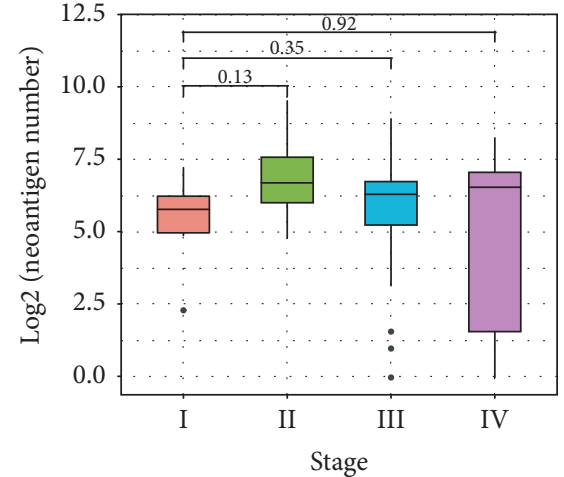

Stage

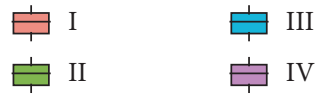

(c)

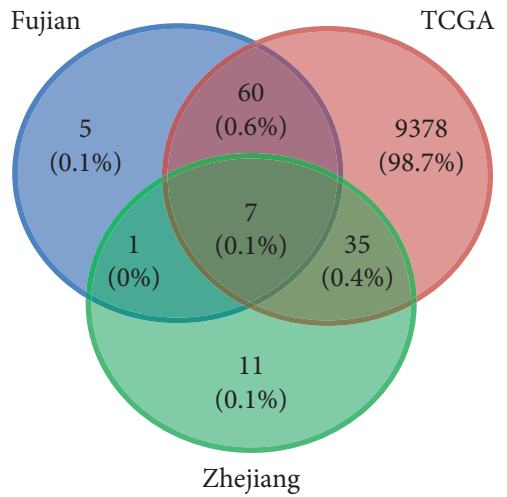

(e)

(f)

FIGURE 3: The comparation of neoantigens between different subgroups and cohorts: (a) between age $\geqq 60$ and age $<60$ groups; (b) between female and male groups; (c) between different stages; (d) between different locations; (e) between different Lauren types; (f) between different cohorts.

respectively). Although not all samples can obtain HLA information, we believe that these neoantigens are also potential tumor-specific neoantigens in other GC samples.

\subsection{Hotspot Mutation-Related Neoantigens That May Be a} Potential Source of Immunotherapy Target in GC and PanCancer. In order to further study the potential significance of these high frequency neoantigens, we focused our attention on the mutation R175H (TP53), H1047R (PIK3CA), and G12D (KRAS) because these sites can not only produce recurrent neoantigens (frequency of more than 4 occurrences in 482 samples) but also have high mutation frequencies in the TCGA pan-cancer cohort.

TP53 is the most common mutated tumor suppressor gene in GC and other cancers, and TP53 R175H mutation is known to be carcinogenic [36], which located in the DNA binding domain of TP53 protein (Figure 4(a)). R175H mutation has a series of adverse effects, such as reducing the activation of the TP53 target, interfering with the activation of wild-type TP53, leading to resistance to apoptosis, reducing genomic stability, and promoting tumorigenesis and cell migration $[37,38]$. In TCGA pan-cancer cohort (almost primary cancer, $n=11,160$ ), the mutation occurred in 162 (1.5\%) individuals. Similarly, the mutation is a hotspot in metastatic cancers. In the MSKIMPACT study of over 10, 000 metastatic cancers [39], the mutation occurred more than $2 \%$ in nine cancers and $7.2 \%$ in esophagogastric cancers (Figure 4(b)). Although $\mathrm{R} 175 \mathrm{H}$ is widespread in many cancers, there is no targeted drug for this mutation. Therefore, we believe that the mutation may be a potential target for $\mathrm{T}$ cell immunotherapy across different cancer types.

PIK3CA mutations are mainly found in EBV-positive GC [16], and it is one of the driver genes in cancer. PIK3CA 
TABle 2: The list of top 10 neoantigens, their corresponding mutation (AA change), neoantigen frequency in Fujian and TCGA cohorts (Freq1, $n=482$ ), mutation frequency in all GC cohort (Freq2, $n=942$ ), and TCGA pan-cancer cohort (Freq3, $n=11,160$ ), and HLA information.

\begin{tabular}{|c|c|c|c|c|c|}
\hline Gene & AA change & Freq1 & Freq2 & Freq3 & HLA types \\
\hline PGM5 & I98V & 10 & 26 & 51 & $\begin{array}{l}\text { HLA-A02:30; HLA-A02:01; HLA-A02:03; HLA-A02:05; HLA- } \\
\text { A02:06; HLA-A02:07; HLA-C03:03 }\end{array}$ \\
\hline TP53 & $\mathrm{R} 175 \mathrm{H}$ & 8 & 25 & 162 & HLA-C07:02; HLA-C07:01; HLA-A68:01; HLA-A02:03 \\
\hline TRIM49C & S327R & 7 & 10 & 17 & $\begin{array}{l}\text { HLA-B35:03; HLA-C06:02; HLA-C12:03; HLA-C07:01; HLA- } \\
\text { C07:02; HLA-B27:08; HLA-B07:02; HLA-A02:01; HLA-B27:05; } \\
\text { HLA-B57:01; HLA-A32:01; HLA-A11:01; HLA-A31:01 }\end{array}$ \\
\hline PIK3CA & H1047R & 5 & 24 & 298 & $\begin{array}{l}\text { HLA-C07:02; HLA-C07:01; HLA-A30:01; HLA-B58:01; HLA- } \\
\text { B57:01; HLA-A33:03; HLA-A68:01 }\end{array}$ \\
\hline TP53 & $\mathrm{R} 273 \mathrm{H}$ & 5 & 16 & 145 & HLA-A02:01; HLA-A02:07; HLA-A02:17; HLA-A68:01 \\
\hline KRAS & G13D & 5 & 15 & 78 & $\begin{array}{l}\text { HLA-A02:01; HLA-A11:01; HLA-A68:01 } \\
\text { HLA-C06:02; HLA-B08:01; HLA-C07:01; HLA-A30:01; HLA- }\end{array}$ \\
\hline$E R B B 3$ & V104M & 5 & 11 & 33 & $\begin{array}{l}\text { C12:03; HLA-B35:01; HLA-A30:02; HLA-A68:01; HLA-B07:02; } \\
\text { HLA-A03:01; HLA-C07:02; HLA-A02:01 }\end{array}$ \\
\hline C6 & K817T & 4 & 5 & 9 & $\begin{array}{l}\text { HLA-C03:04; HLA-C07:01; HLA-C03:03; HLA-C12:02; HLA- } \\
\text { A02:01; HLA-C02:10; HLA-A24:02; HLA-B35:01; HLA-B15:03; } \\
\text { HLA-A31:01 }\end{array}$ \\
\hline KRAS & G12D & 4 & 16 & 430 & $\begin{array}{l}\text { HLA-A02:01; HLA-C05:01; HLA-A02:06; HLA-A11:01; HLA- } \\
\text { A03:01; HLA-B07:02 }\end{array}$ \\
\hline TP53 & $\mathrm{R} 282 \mathrm{~W}$ & 4 & 14 & 97 & $\begin{array}{l}\text { HLA-C03:03; HLA-A11:01; HLA-A03:01; HLA-B07:02; HLA- } \\
\text { A68:01 }\end{array}$ \\
\hline OR4C16 & S135R & 4 & 6 & 8 & $\begin{array}{l}\text { HLA-B37:01; HLA-A02:06; HLA-A02:01; HLA-B15:01; HLA- } \\
\text { C03:03; HLA-B27:05; HLA-A03:01; HLA-C07:02; HLA-A24:02 }\end{array}$ \\
\hline
\end{tabular}

H1047R is a gain-of-function mutation located in the kinase region (Figure $4(\mathrm{c})$ ), and it is oncogenic and most common in breast cancer $[40,41]$. The frequency of this mutation in TCGA pan-cancer was as high as 298 (2.7\%), and it was more than $2 \%$ in multiple metastatic cancer types in the MSK-IMPACT cohort (Figure 4(d)). PIK3CA gene has many mutation forms at this site (including H1047R, H1047Y, and H1047L). Zhou et al. reported that H1047Y mutation can produce tumor-specific neoantigen in a GC patient [21]. However, it is worth noting that H1047R mutation is the main mutation form of PIK3CA, whether in TCGA GC cohort or MSK pan-cancer cohort. For breast cancer patients carrying the mutation, there are targeted drugs for the mutation, such as Alpelisib and Fulvestrant [42]. As mentioned earlier, PIK3CA mutation and TP53 mutation are exclusive in GC, so for TP53 mutation negative patients, T cell immunotherapy based on PIK3CA H1047R may be an option, and it is also a potential choice for breast cancer or other cancer types that are resistant to targeted drugs. In addition, KRAS Gly12 (including G12V, G12C, and G12D) is also a classic cancer mutation, and the mutation frequency of this site in metastatic pancreatic and appendiceal cancers is more than 20\% [39]. Both Charoentong and Witkiewicz have reported the high frequency of the KRAS G12D mutation in pancreatic cancer $[20,43]$. In fact, the first clinical trial to test this immunotherapy regimen in HLA-A11:01 cancer patients with KRAS G12V mutation has begun (NCT03190941).

\section{Discussion}

Before this study, Zhou et al. had published a neoantigen prediction study for GC. However, there are some limitations in their research, which have been solved in this study. Firstly, their study only involved 32 samples of GC, and no neoantigens were found shared among GC patients. Secondly, they only used a neoantigen prediction software, several neoantigen prediction software have been released and updated recently [29-33], and research shows that the combination of multiple software can better improve the accuracy and sensitivity of neoantigen prediction [32]. Thirdly, we found several neoantigens associated mutation significantly mutated in GC patients or pan-cancer cohort, such as PIK3CA H1047R and TP53 R175H, which were not found in previous studies. Finally, mutants of MUC4 were reported in 94\% (30/32) of patients in the Zhejiang cohort, but previous studies in several GC cohorts have never found such a high mutation frequency of the gene MUC4 $[14-19,34,35]$. It seems that their study has obvious falsepositive results in mutation detection, and the accuracy of mutation detection results will directly affect the predictive results of neoantigens.

For the recurrent neoantigens identified in this study, there are a variety of HLA alleles that can present them to the cell surface (Table 2). Among them, HLA-A11:01, HLA-A24:02, HLA-A02:01, and HLA-C07:02 mainly appeared in the Chinese population [44], indicating that cancer patients with these HLA-type in China will be potential therapeutic populations for these "public" neoantigens. Of course, there is still a long way to go for the immunotherapy of these "public" neoantigens. Firstly, these neoantigens should be experimentally confirmed to be presented and recognized by $\mathrm{T}$ cells. Secondly, it is necessary to ensure that normal tissue cells do not express these antigens in order to avoid the adverse autoimmune reactions of $\mathrm{T}$ cells attacking normal tissues. 


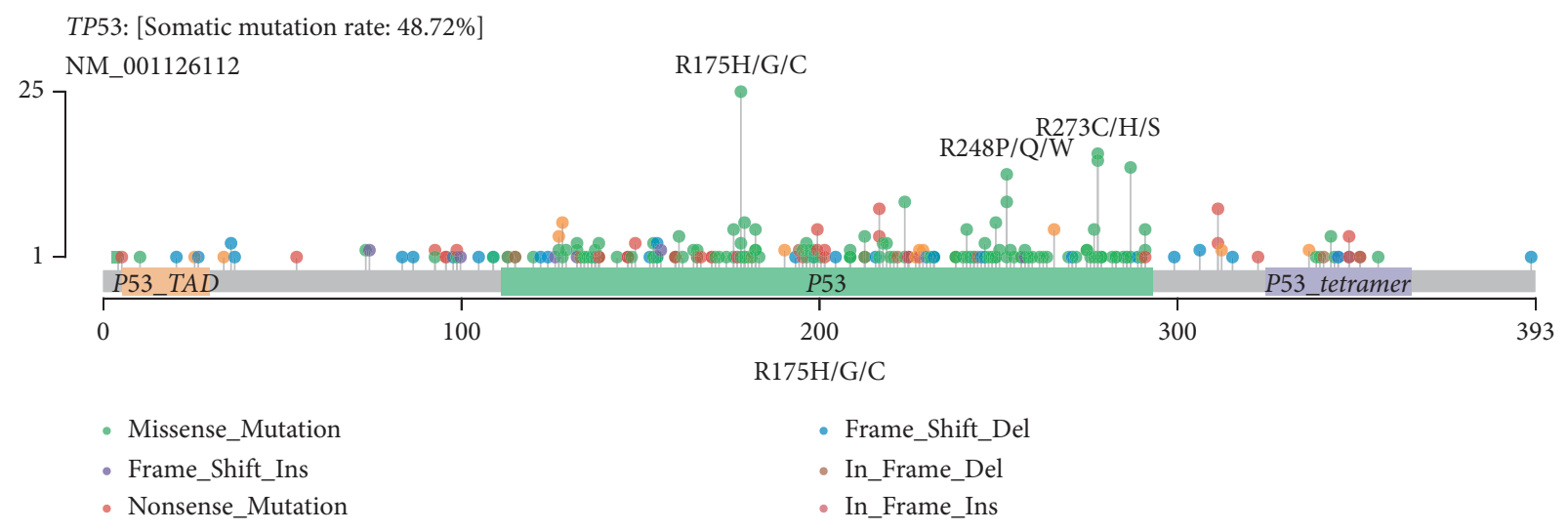

- Splice_Site

(a)

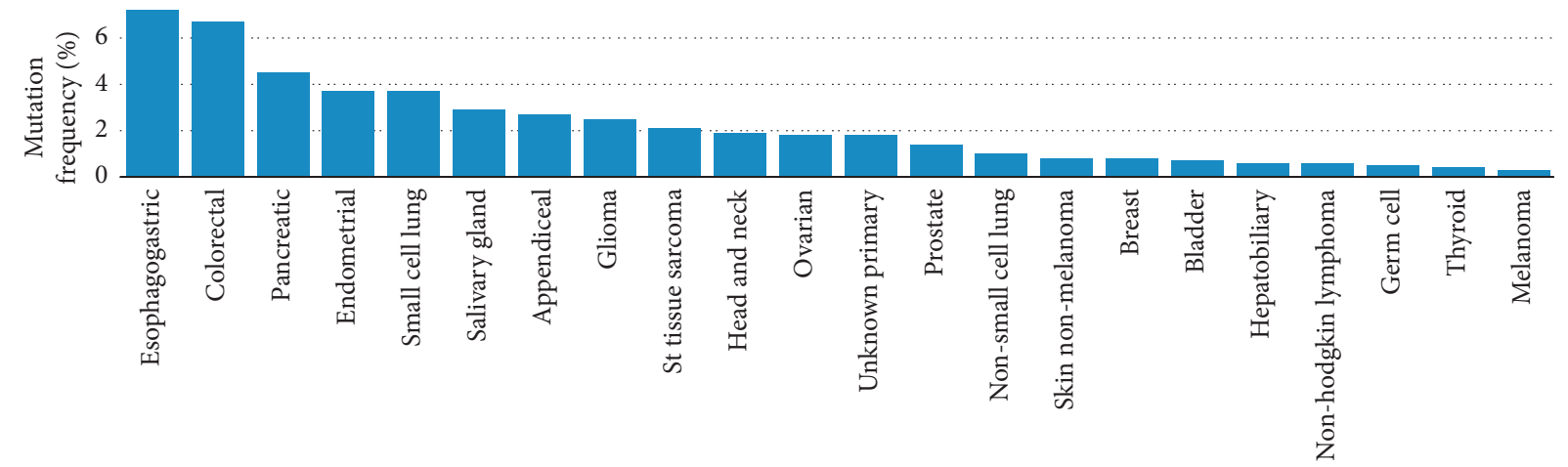

(b)

PIK3CA: [Somatic mutation rate: $10.81 \%$ ]

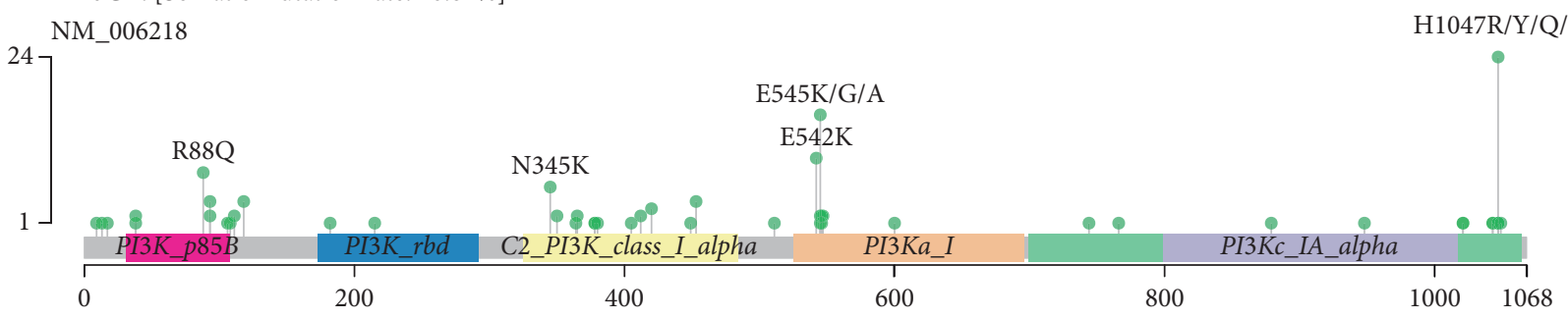

— Missense_Mutation

(c)

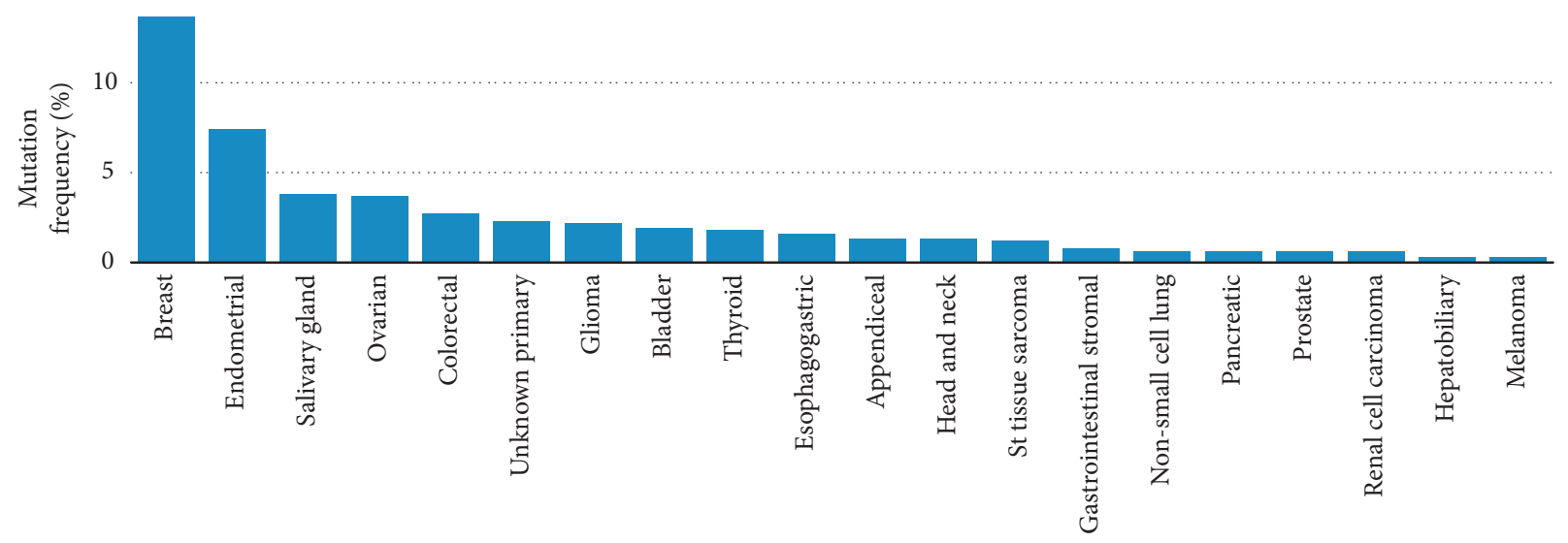

(d)

FIGURE 4: TP53 mutational spectrum in 942 GC patients (a) and TP53 R175H mutation in MSK-IMPACT pan-cancer cohorts (b). PIK3CA mutational spectrum in 942 GC patients (c) and PIK3CA H1047R mutation in MSK-IMPACT pan-cancer cohorts (d). 
These can be accomplished with the help of highly sensitive mass spectrometry-based assay and a series of experiments [45].

In this study, in order to promote the immunotherapy of GC based on neoantigens, we combined 74 samples of Fujian cohort with published sequencing data to obtain 942 samples and carried out the largest study of the mutation and neoantigen landscape of GC so far. Firstly, we constructed the most complete mutational profiling of GC so far. Notably, we found five genes were mutated exclusively with TP53, including CDH1, KMT2D, RYR1, $P I K 3 C A$, and ARID1A (Fisher's exact test, $P<0.05$, Figure S2), which four of them (CDH1, KMT2D, RYR1, and PIK3CA) were not reported before. Secondly, we use a combination of multiple software to detect neoantigens, avoiding the limitations of a single software (for example, PSSMHCpan [32] can pan-specifically predict peptides that bind to 4896 HLA class I alleles, while NetMHC-4.0 [29] and NetMHCpan-3.0 [30] can predict only 89 and 2924 HLA class I alleles, respectively) [32]. Thirdly, the purpose of this study is not only to obtain the neoantigens of GC but also to find potential common antigens in GC patients and to promote the immunotherapy of GC. By combining Fujian and TCGA cohorts, 482 GC samples were predicted for neoantigens, and 549 neoantigens were found to be shared among the samples. If we consider the top ten neoantigens as a panel, it can cover about $15.8 \%$ of the total 482 samples. At present, 10-20 cancer-specific neoantigens are usually synthesized in vitro and used in T cell immunotherapy [11]. Since these neoantigens can cover a certain proportion of GC population, we believe that these recurrent neoantigens of GC have potential clinical application value. It is believed that, with the development of neoantigen identification technology, more kinds and more quantities of neoantigens will be discovered, such as those produced by tumor-specific expressed abnormal splicing transcripts from noncoding regions of human genome [46], neoantigen-based immunotherapy will play an increasingly important role in cancer treatment.

\section{Conclusions}

Overall, based on 942 whole exome/genome sequencing data of Fujian samples and other published data, the most complete mutation landscape of GC was obtained. Based on the mutation data and HLA information, several recurrent neoantigen-associated mutations, such as PIK3CA H1047R and TP53 R175H, were predicted. Some of these neoantigenassociated mutations also have high frequencies in pancancer, indicating that they are potential targets for pancancer immunotherapy.

\section{Data Availability}

The data reported in this study are available in the CNGB Nucleotide Sequence Archive (CNSA: https://db.cngb.org/ cnsa;accession number CNP0000159). Other data can be obtained by contacting the corresponding author.

\section{Conflicts of Interest}

The authors declare that there are no conflicts of interest regarding the publication of this paper.

\section{Authors' Contributions}

Chao Chen, Qiming Zhou, and Riping Wu contributed equally.

\section{Acknowledgments}

We thank the department of pathology at Union Medical College Hospital Affiliated to Fujian Medical University for its help in sample and data collection. We also thank Michael Dean for his constructive comments on the manuscript. This work was supported by the Critical Patented Project of the Science \& Technology Bureau of Fujian Province, China, under grant no. 2013YZ0002-2, the Joint Project of the Natural Science and Health Foundation of Fujian Province, China, under grant no. 2015J01397, Science, Technology and Innovation Commission of Shenzhen Municipality under grant no. GJHZ20170314152701465, and the Shenzhen Science and Technology Program under grant no. JCYJ20170817145845968.

\section{Supplementary Materials}

Supplementary Figure S1: mutational landscape of the 942 GC (gastric cancer) samples. Supplementary Figure S2: somatic mutation interactions between genes. Supplementary Table S1: six published GC genomic literature involving 942 GC samples in this study. Supplementary Table S2: clinical information of 942 GC samples. Supplementary Table S3: mutation sites, frequencies, and HLA I alleles of all predicted neoantigens. (Supplementary Materials)

\section{References}

[1] Y. Kiyozumi, M. Iwatsuki, K. Yamashita, Y. Koga, N. Yoshida, and H. Baba, "Update on targeted therapy and immune therapy for gastric cancer, 2018," Journal of Cancer Metastasis and Treatment, vol. 4, no. 6, p. 31, 2018.

[2] H. Ashktorab, S. S. Kupfer, H. Brim, and J. M. Carethers, "Racial disparity in gastrointestinal cancer risk," Gastroenterology, vol. 153, no. 4, pp. 910-923, 2017.

[3] X. Li, B. Pasche, W. Zhang, and K. Chen, "Association of MUC16 mutation with tumor mutation load and outcomes in patients with gastric cancer," JAMA Oncology, vol. 4, no. 12, pp. 1691-1698, 2018.

[4] N. Zacharakis, H. Chinnasamy, M. Black et al., "Immune recognition of somatic mutations leading to complete durable regression in metastatic breast cancer," Nature Medicine, vol. 24, no. 6, pp. 724-730, 2018.

[5] C. J. Cohen, J. J. Gartner, M. Horovitz-Fried et al., "Isolation of neoantigen-specific $\mathrm{T}$ cells from tumor and peripheral lymphocytes," Journal of Clinical Investigation, vol. 125, no. 10, pp. 3981-3991, 2015.

[6] Q. J. Wang, Z. Yu, K. Griffith, K.-I. Hanada, N. P. Restifo, and J. C. Yang, "Identification of T-cell receptors targeting KRASmutated human tumors," Cancer Immunology Research, vol. 4, no. 3, pp. 204-214, 2016. 
[7] C. A. Klebanoff, S. A. Rosenberg, and N. P. Restifo, "Prospects for gene-engineered T cell immunotherapy for solid cancers," Nature Medicine, vol. 22, no. 1, pp. 26-36, 2016.

[8] P. F. Robbins, Y.-C. Lu, M. El-Gamil et al., "Mining exomic sequencing data to identify mutated antigens recognized by adoptively transferred tumor-reactive T cells," Nature Medicine, vol. 19, no. 6, pp. 747-752, 2013.

[9] T. N. Samuels and R. D. Schreiber, "Neoantigens in cancer immunotherapy," Science, vol. 348, no. 6230, pp. 69-74, 2015.

[10] B. M. Carreno, V. Magrini, M. Becker-Hapak et al., "A dendritic cell vaccine increases the breadth and diversity of melanoma neoantigen-specific T cells," Science, vol. 348, no. 6236, pp. 803-808, 2015.

[11] P. A. Ott, Z. Hu, D. B. Keskin et al., "An immunogenic personal neoantigen vaccine for patients with melanoma," Nature, vol. 547, no. 7662, pp. 217-221, 2017.

[12] Z. S. Chheda, G. Kohanbash, K. Okada et al., "Novel and shared neoantigen derived from histone 3 variant $\mathrm{H} 3.3 \mathrm{~K} 27 \mathrm{M}$ mutation for glioma T cell therapy," The Journal of Experimental Medicine, vol. 215, no. 1, pp. 141-157, 2018.

[13] S. Bobisse, R. Genolet, A. Roberti et al., "Sensitive and frequent identification of high avidity neo-epitope specific CD8 (+) T cells in immunotherapy-naive ovarian cancer," Nature Communications, vol. 9, no. 1, p. 1092, 2018.

[14] R. Xing, Y. Zhou, J. Yu et al., "Whole-genome sequencing reveals novel tandem-duplication hotspots and a prognostic mutational signature in gastric cancer," Nature Communications, vol. 10, no. 1, p. 2037, 2019.

[15] K. Chen, D. Yang, X. Li et al., "Mutational landscape of gastric adenocarcinoma in Chinese: implications for prognosis and therapy," Proceedings of the National Academy of Sciences, vol. 112, no. 4, pp. 1107-1112, 2015.

[16] The Cancer Genome Atlas Research Network, "Comprehensive molecular characterization of gastric adenocarcinoma," Nature, vol. 513, no. 7517, pp. 202-209, 2014.

[17] S. S. Wong, K. M. Kim, J. C. Ting et al., "Genomic landscape and genetic heterogeneity in gastric adenocarcinoma revealed by whole-genome sequencing," Nature Communications, vol. 5, no. 1, p. 5477, 2014.

[18] M. Kakiuchi, T. Nishizawa, H. Ueda et al., "Recurrent gain-offunction mutations of RHOA in diffuse-type gastric carcinoma," Nature Genetics, vol. 46, no. 6, pp. 583-587, 2014.

[19] K. Wang, S. T. Yuen, J. Xu et al., "Whole-genome sequencing and comprehensive molecular profiling identify new driver mutations in gastric cancer," Nature Genetics, vol. 46, no. 6 , pp. 573-582, 2014.

[20] P. Charoentong, F. Finotello, M. Angelova et al., "Pan-cancer immunogenomic analyses reveal genotype-immunophenotype relationships and predictors of response to checkpoint blockade," Cell Reports, vol. 18, no. 1, pp. 248-262, 2017.

[21] J. Zhou, W. Zhao, J. Wu et al., "Neoantigens derived from recurrently mutated genes as potential immunotherapy targets for gastric cancer," BioMed Research International, vol. 2019, Article ID 8103142, 11 pages, 2019.

[22] Y. Chen, Y. Chen, C. Shi et al., "SOAPnuke: a MapReduce acceleration-supported software for integrated quality control and preprocessing of high-throughput sequencing data," GigaScience, vol. 7, no. 1, pp. 1-6, 2018.

[23] K. Cibulskis, M. S. Lawrence, S. L. Carter et al., "Sensitive detection of somatic point mutations in impure and heterogeneous cancer samples," Nature Biotechnology, vol. 31, no. 3, pp. 213-219, 2013.

[24] D. C. Koboldt, Q. Zhang, D. E. Larson et al., "VarScan 2: somatic mutation and copy number alteration discovery in cancer by exome sequencing," Genome Research, vol. 22, no. 3, pp. 568-576, 2012.

[25] K. Wang, M. Li, and H. Hakonarson, "ANNOVAR: functional annotation of genetic variants from high-throughput sequencing data," Nucleic Acids Research, vol. 38, no. 16, p. e164, 2010.

[26] A. Mayakonda, D.-C. Lin, Y. Assenov, C. Plass, and H. P. Koeffler, "Maftools: efficient and comprehensive analysis of somatic variants in cancer," Genome Research, vol. 28, no. 11, pp. 1747-1756, 2018.

[27] C. Chen, C. Shi, X. Huang et al., "Molecular profiles and metastasis markers in Chinese patients with gastric carcinoma," Scientific Reports, vol. 9, no. 1, 2019.

[28] S. A. Shukla, M. S. Rooney, M. Rajasagi et al., "Comprehensive analysis of cancer-associated somatic mutations in class I HLA genes," Nature Biotechnology, vol. 33, no. 11, pp. 1152-1158, 2015.

[29] C. Lundegaard, K. Lamberth, M. Harndahl, S. Buus, O. Lund, and M. Nielsen, "NetMHC-3.0: accurate web accessible predictions of human, mouse and monkey MHC class I affinities for peptides of length 8-11," Nucleic Acids Research, vol. 36, no. 2, pp. W509-W512, 2008.

[30] I. Hoof, B. Peters, J. Sidney et al., "NetMHCpan, a method for MHC class I binding prediction beyond humans," Immunogenetics, vol. 61, no. 1, pp. 1-13, 2009.

[31] H. Zhang, O. Lund, and M. Nielsen, "The pickpocket method for predicting binding specificities for receptors based on receptor pocket similarities: application to MHC-peptide binding," Bioinformatics, vol. 25, no. 10, pp. 1293-1299, 2009.

[32] G. Liu, D. Li, Z. Li et al., "PSSMHCpan: a novel PSSM-based software for predicting class I peptide-HLA binding affinity," GigaScience, vol. 6, no. 5, pp. 1-11, 2017.

[33] B. Peters and A. Sette, "Generating quantitative models describing the sequence specificity of biological processes with the stabilized matrix method," BMC Bioinformatics, vol. 6, no. 1, p. 132, 2005.

[34] Z. J. Zang, I. Cutcutache, S. L. Poon et al., "Exome sequencing of gastric adenocarcinoma identifies recurrent somatic mutations in cell adhesion and chromatin remodeling genes," Nature Genetics, vol. 44, no. 5, pp. 570-574, 2012.

[35] K. Wang, J. Kan, S. T. Yuen et al., "Exome sequencing identifies frequent mutation of ARID1A in molecular subtypes of gastric cancer," Nature Genetics, vol. 43, no. 12, pp. 1219-1223, 2011.

[36] W. A. Freed-Pastor and C. Prives, "Mutant p53: one name, many proteins," Genes \& Development, vol. 26, no. 12, pp. 1268-1286, 2012.

[37] W. A. Yeudall, C. A. Vaughan, H. Miyazaki et al., "Gain-offunction mutant p53 upregulates CXC chemokines and enhances cell migration," Carcinogenesis, vol. 33, no. 2, pp. 442-451, 2012.

[38] D. P. Liu, H. Song, and Y. Xu, "A common gain of function of p53 cancer mutants in inducing genetic instability," Oncogene, vol. 29, no. 7, pp. 949-956, 2010.

[39] A. Zehir, R. Benayed, R. H. Shah et al., "Mutational landscape of metastatic cancer revealed from prospective clinical sequencing of 10,000 patients," Nature Medicine, vol. 23, no. 6, pp. 703-713, 2017.

[40] J. A. Engelman, L. Chen, X. Tan et al., "Effective use of PI3K and MEK inhibitors to treat mutant Kras G12D and PIK3CA H1047R murine lung cancers," Nature Medicine, vol. 14, no. 12, pp. 1351-1356, 2008.

[41] S. J. Isakoff, J. A. Engelman, H. Y. Irie et al., "Breast cancerassociatedpik3camutations are oncogenic in mammary 
epithelial cells," Cancer Research, vol. 65, no. 23, pp. 1099211000, 2005.

[42] D. Juric, F. Janku, J. Rodon et al., "Alpelisib plus fulvestrant in PIK3ca-altered and PIK3ca-wild-type estrogen receptorpositive advanced breast cancer: a phase $1 \mathrm{~b}$ clinical trial," JAMA Oncology, vol. 5, no. 2, Article ID e184475, 2019.

[43] A. K. Witkiewicz, E. A. McMillan, U. Balaji et al., "Wholeexome sequencing of pancreatic cancer defines genetic diversity and therapeutic targets," Nature Communications, vol. 6, no. 1, 6744 pages, 2015

[44] F. Zhou, H. Cao, X. Zuo et al., "Deep sequencing of the MHC region in the Chinese population contributes to studies of complex disease," Nature Genetics, vol. 48, no. 7, pp. 740-746, 2016.

[45] M. Jin, E. Braunlein, R. Klar et al., "Direct identification of clinically relevant neoepitopes presented on native human melanoma tissue by mass spectrometry," Nature Communications, vol. 7, no. 1, 13404 pages, 2016.

[46] C. M. Laumont, K. Vincent, L. Hesnard et al., "Noncoding regions are the main source of targetable tumor-specific antigens," Science Translational Medicine, vol. 10, no. 470, Article ID eaau5516, 2018. 


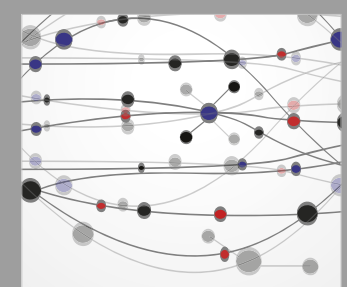

The Scientific World Journal
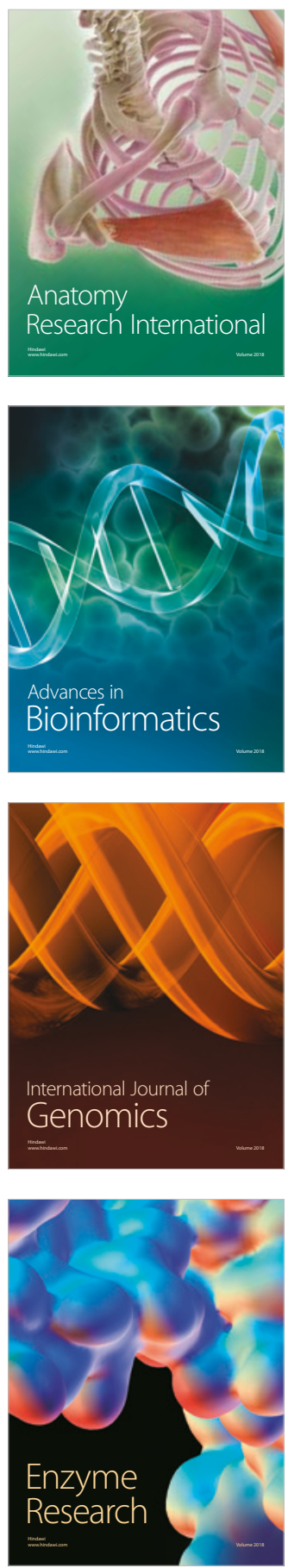
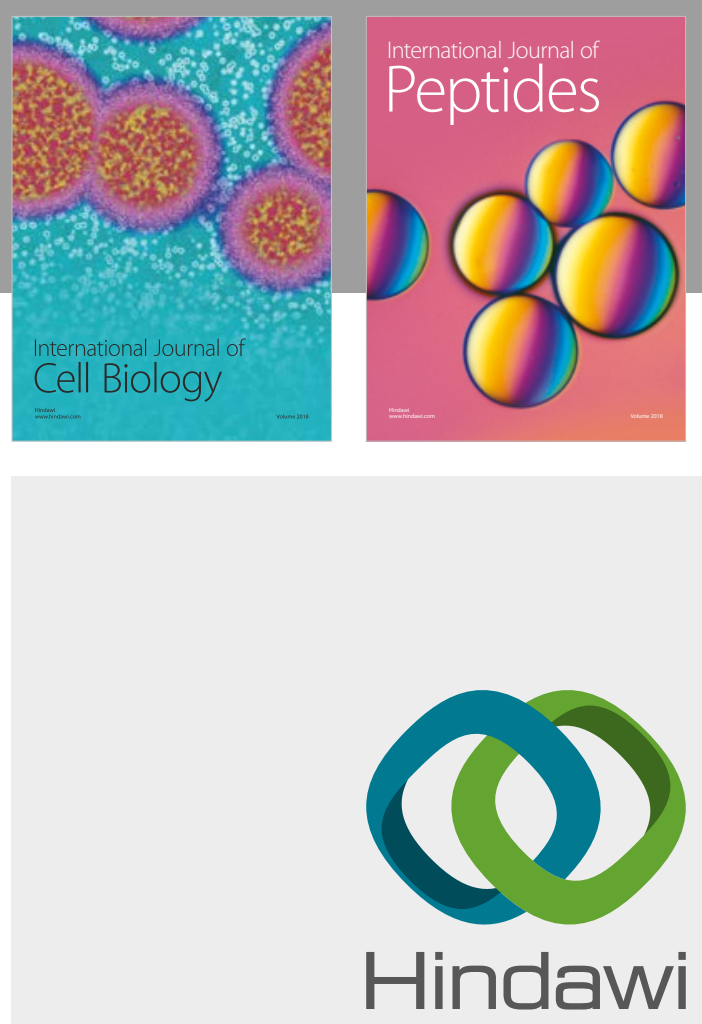

Submit your manuscripts at

www.hindawi.com
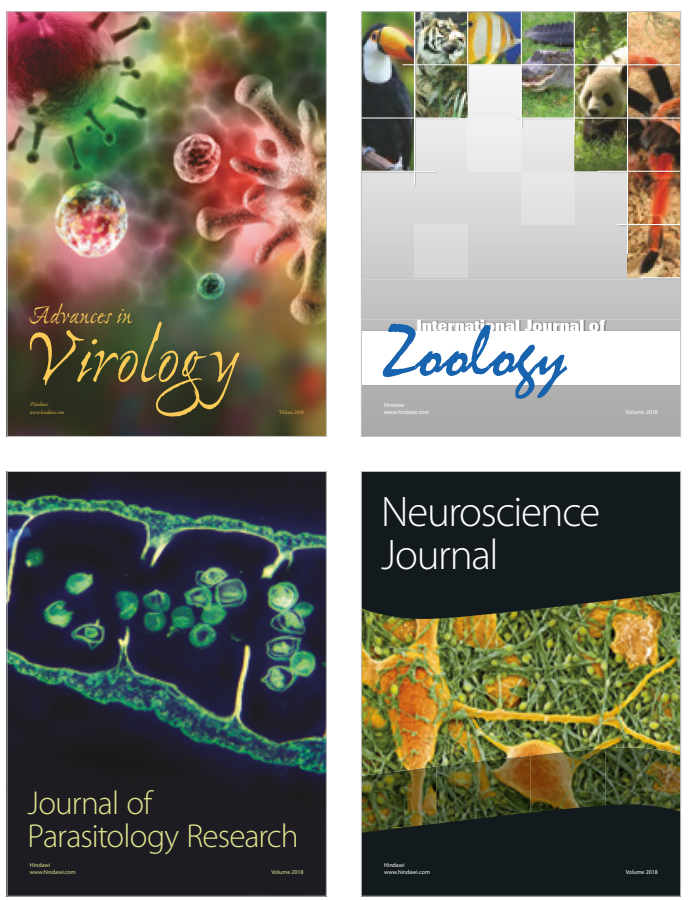
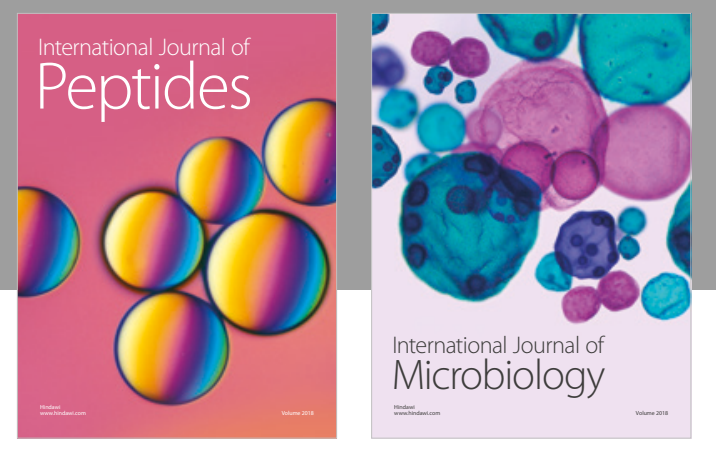

nternational Journal of Microbiology
Journal of
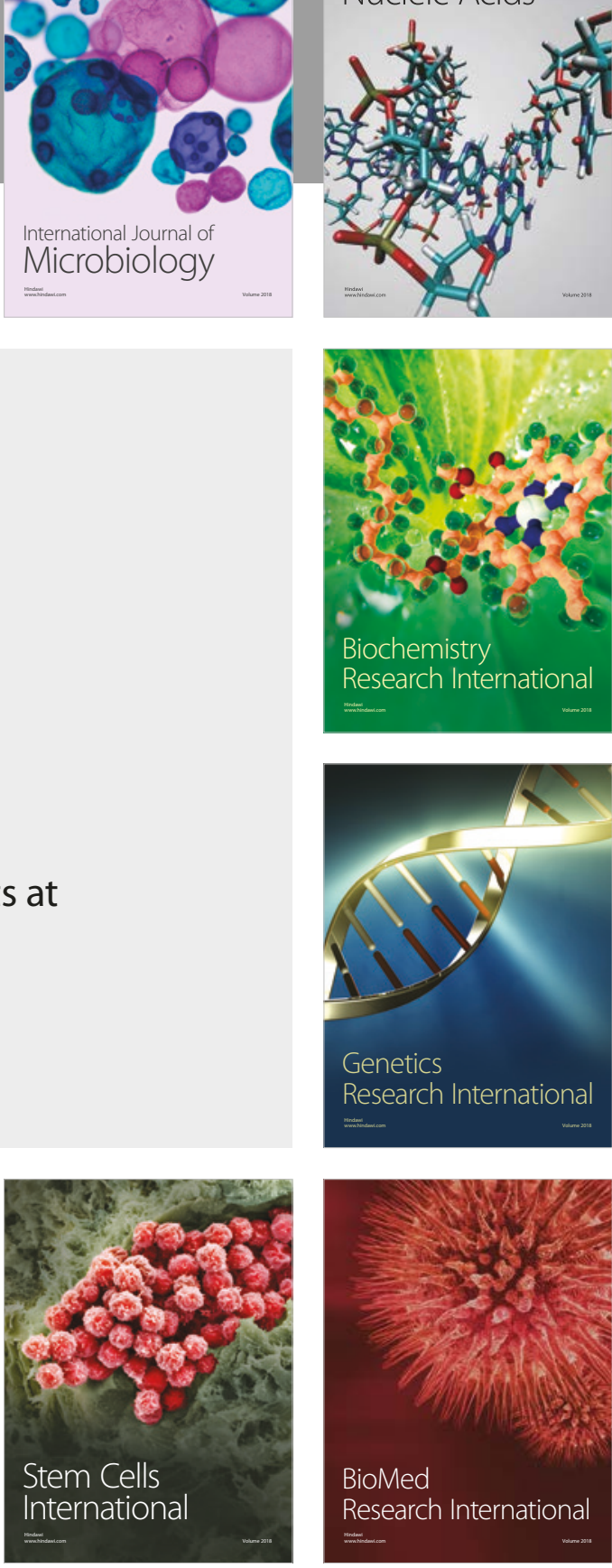
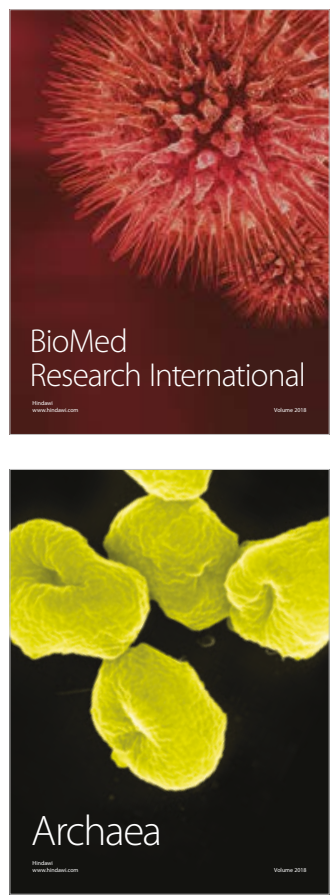